\title{
Spectral properties of a partially spin-polarized one-dimensional Hubbard/Luttinger superfluid
}

\author{
Adrian E. Feiguin ${ }^{1}$ and David A. Huse ${ }^{2}$ \\ ${ }^{I}$ Microsoft Station Q, University of California, Santa Barbara, California 93106, USA \\ ${ }^{2}$ Department of Physics, Princeton University, New Jersey 08544, USA
}

(Dated: October 26, 2018)

\begin{abstract}
We calculate the excitation spectra of a spin-polarized Hubbard chain away from half-filling, using a highprecision momentum-resolved time-dependent Density Matrix Renormalization Group method. Focusing on the $U<0$ case, we present in some detail the single-fermion, pair, density and spin spectra, and discuss how spincharge separation is altered for this system. The pair spectra show a quasi-condensate at a nonzero momentum proportional to the polarization, as expected for this Fulde-Ferrel-Larkin-Ovchinnikov-like superfluid.
\end{abstract}

PACS numbers:

Systems of interacting fermions obeying Fermi liquid theory exhibit a one-to-one correspondence between their lowenergy quasiparticle excitations and those of a non-interacting Fermi gas. The quasiparticles have renormalized energy and spectral weight, but possess the same charge and spin quantum numbers as the corresponding noninteracting fermions. This scenario breaks down in one dimension (1D): in this case, each Fermi surface reduces to two points in momentum space, at $k= \pm k_{F}$. The resulting Fermi-surface nesting is present at all densities and spin polarizations and destabilizes the Fermi liquid, converting it instead to a Luttinger liquid [1, 2, 3], even for a weak interaction.

In a Luttinger liquid with zero spin polarization, the elementary excitations are collective density fluctuations that carry only either spin ("spinons") or charge ("holons"). These excitations have different dispersions, and, obviously, do not carry the same quantum numbers as the original "bare" fermions. This leads to the spin-charge separation picture, in which a fermion injected into the system separates ("fractionates") into an (anti-)holon and a spinon, each of them carrying a share of the fermion's quantum numbers. The phenomenon of spin-charge separation, and fractionation of particles more generally, is an important concept in strongly-correlated systems, and has intrigued physicists for decades. Its signatures have been observed experimentally in 1D organic conductors [4], metallic wires [5], carbon nanotubes [6], and nanowires in semiconducting heterostructures [7]. Proposals have been made to seek for evidence of these phenomena in cold atomic gases [8, 9].

In the 1D Hubbard model, the low-energy spin and charge modes of the Luttinger liquid decouple as long as the system either is at half-filling or has zero spin-polarization. However, if the system is away from half-filling and has a nonzero magnetization, the collective modes that constitute the elementary low-lying excitations are linear combinations of the spin and charge fields [10, 11, 12], so although one still has a Luttinger liquid with fractionalized fermions, it is no longer strictly a "spin-charge separation" scenario. The field-theoretical formulation of the Luttinger liquid theory has proven very effective in describing the low-energy physics of a variety of models. However, a fully quantitative and general picture of how the spin and charge degrees of freedom couple to form full-fledged fermions is still missing.

In this work, we will focus our attention on the negative$U$ (attractive) 1D Hubbard model, away from half-filling and at nonzero spin polarization. This model can now be studied experimentally with ultracold atoms in an optical lattice. As usual, the Hamiltonian is

$$
H=-t \sum_{i, \sigma}\left(c_{i \sigma}^{\dagger} c_{i+1 \sigma}+\text { h.c. }\right)+U \sum_{i} n_{i \uparrow} n_{i \downarrow},
$$

where $c_{\ell \sigma}^{\dagger}$ creates a fermion with spin $\sigma=\uparrow, \downarrow$ at site $\ell$; $n_{\ell \sigma}=c_{\ell \sigma}^{\dagger} c_{\ell \sigma} ; t$ is the hopping matrix element, which we set to unity (we also set the lattice spacing to unity); and $U$ is the interaction strength that in this work will be considered negative (attractive). The negative and positive $U$ versions of this model can be mapped exactly onto each other by the "canonical" transformation that applies a particle-hole and momentum change to one spin species. Thus our results are general, and can be translated to the positive- $U$ case [13].

For large negative $U$ the fermions form tightly bound pairs that behave as hard-core bosons [13]. These bosons are prevented from fully condensing in 1D due to quantum fluctuations. They form a "quasi-condensate", with pair correlations that decay as a power-law that, in some regime of parameters (large $|U|>4 t$ ) can dominate over the single-fermion correlations at large distances [14]. In the polarized case, the ground-state of this system is the 1D version of the FuldeFerrell-Larkin-Ovchinnikov (FFLO) superfluid [14, 15, 16], in which the pairs forming the quasi-condensate have nonzero center of mass momentum $\pm Q$ with $Q=k_{F \uparrow}-k_{F \downarrow}$, where $k_{F \sigma}$ is the Fermi momentum of the fermions with spin $\sigma$. This was confirmed numerically in Ref.[17], and subsequent studies [18, 19]).

The Luttinger-liquid and FFLO aspects of this system can be heuristically understood as follows: At large negative $U$, the spin-polarized ground state consists of empty sites ( 0 's), sites occupied by pairs (2's) and excess up fermions ( $\uparrow$ 's), with sites singly-occupied by $\downarrow$ 's being only "virtual" states. The density of excess $\uparrow$ 's is $Q / \pi$. An $\uparrow$ exchanges positions with the 0's and 2's with hopping $t$ and thus moves with bandwidth $4 t$. At half-filling, the background the $\uparrow$ moves through is half 0 's and half 2's, so the relative motion moves spin but no den- 
sity on average: this collective mode is then purely a spinon. But if we move away from half-filling, the number of 0's differs from the number of 2's, so when an $\uparrow$ moves, it on average moves some density as well as spin: this light (bandwidth $4 t$ ) mode of the Luttinger liquid is then not purely spin, but instead is a particular linear combination of spin and charge (we will call this light mode "spinon-like"). In the limits of nearly complete polarization or either zero or complete filling, the $\uparrow$ 's become just regular fermions carrying the full charge and spin. This scenario has been confirmed numerically in Ref. $[12,20,21]$, by looking at the real-time evolution of spin and charge distributions.

At large negative $U$ the 2's do not move freely past the 0 's; this exchange happens via a virtual intermediate unpaired state with energy $|U|$, resulting in effective hopping $t_{\text {eff }}=-2 t^{2} / U$. Thus this motion of 2's relative to 0's constitutes the heavy "holon-like" mode of the Luttinger liquid with a smaller bandwidth. Also, when a 2 moves past an $\uparrow$, the ground state has a sign change. This means the wavefunction of the quasi-condensate of bosonic 2's has a node at each $\uparrow$. If these nodes were equally-spaced, this would be an FFLO standing-wave condensate with momentum $\pm Q$. However, the $\uparrow$ 's actually form a 1D Luttinger liquid with divergent position fluctuations, so the momentum distribution of the pairs instead has a power-law divergence at $\pm Q$; this 1D partially spin-polarized superfluid state should perhaps be termed "quasi-FFLO".

The Hamiltonian (1) can be solved exactly by means of the Bethe Ansatz [22, 23, 24], and the dispersion of the elementary excitations can be obtained [25, 26, 27]. However, the actual Green's functions and spectral properties can only be calculated in certain limits [28], and numerical methods have been crucial to fill in the blanks and compare to experiments [29, 30]. In the following, we use the time-dependent extension of the Density Matrix Renormalization Group (tDMRG) [31, 32] method to obtain estimates for various Green functions in real-time and real-space with unprecedented accuracy [33]. To extract the dynamical response of the system, we calculate the correlators $G\left(x-x^{\prime}, t^{\prime}-t\right)=i\left\langle O\left(x^{\prime}, t^{\prime}\right) O^{\dagger}(x, t)\right\rangle$, where $O$ is an operator of interest. Fourier transforming then yields the corresponding spectral weights as functions of momentum and frequency [31, 33, 34]:

$$
I(k, \omega)=\sum_{n}\left|\left\langle\psi_{n}\left|O_{k}\right| \psi_{0}\right\rangle\right|^{2} \delta\left(\omega-E_{n}+E_{0}\right),
$$

where $E_{0}$ is the ground state energy, and the sum runs over all the eigenstates of the system, with energy $E_{n}$. All the results will be plotted using a log-scale for the intensity, with several orders of magnitude between the intensities of the weakest and strongest features. At very small scales, some ripples or oscillations appear as a consequence of the numerical Fourier transform, and the commensuration of the lattice. These effects get amplified near zero momentum and frequency.

In Fig. 1 (a) and (b) we show the dynamic structure factor for the charge and spin densities, respectively, for an unpolarized Hubbard chain at quarter-filling (in this paper we always
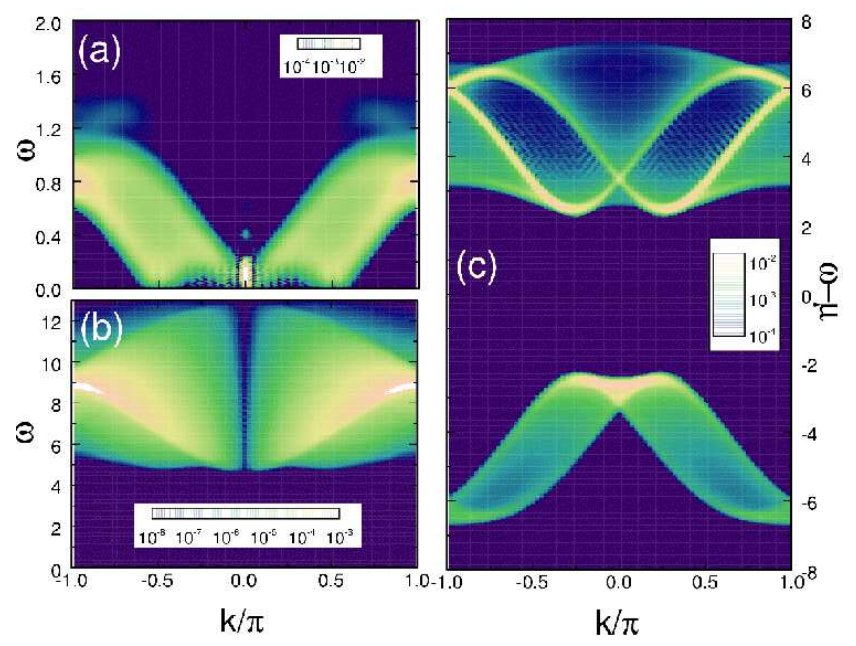

FIG. 1: (color online) Dynamical structure factors of the (a) "charge" density $n(k, \omega)$, and (b) spin $S_{z}(k, \omega)$ for an unpolarized, quarter-filled Hubbard chain with $U=-8 t$. (c) Spectral weights for adding $(\omega>\mu)$ or removing $(\omega<\mu)$ a fermion for the same system; $\mu$ is the chemical potential. Frequencies are in units of the hopping $t=1$. The colors are set by the logarithm of the spectral intensity.

use $L=80$ and $U=-8 t$ ). The charge excitations display gapless modes at momenta $k=0$ and $k= \pm 2 k_{F}= \pm \pi / 2$, and a continuum ranging from $\omega=0$ to $\omega \cong t=4 t_{\text {eff }}$. This spectrum is formed primarily by holon-antiholon excitations. It is qualitatively similar to the particle-hole spectrum of the corresponding non-interacting system, but with a reduced bandwidth. However, this system is a superfluid with a spin gap of $\cong 5 t$, as is seen in the spectral weight of the spin (Fig. $1 b)$; this is the energy "cost" of breaking a Cooper pair. The spinon has band-width $\cong 4 t$, and the spectral weight of $S_{z}$ vanishes strongly as $k \rightarrow 0$, since the total spin is conserved and the matrix element for making spin excitations thus vanishes at zero momentum.

The single-particle spectral weight for the quarter-filled, unpolarized system is shown in Fig. 11(c), where we plot the imaginary part of the one-particle Green's function. The upper and lower features, for positive and negative frequencies, correspond to the inverse photoemission (IPES) and photoemission (PES) spectra, resulting from adding or removing a fermion, respectively. We have shifted the energies relative to the chemical potential $\mu=\left[E_{0}(N+1)-E_{0}(N-1)\right] / 2$, which lies in the center of the gap for this unpolarized system. This gap is a manifestation of the spin gap due to Cooper pairing: the ground state is a total spin singlet with all fermions paired. The added fermion has no "partner" to pair with, while removing a fermion requires breaking an existing pair, so both processes are gapped.

Again, we can heuristically understand many features of these spectra using the large-negative- $U$ description discussed above. The unpolarized ground state is a quasi-condensate of 2's that form a Luttinger liquid of repulsively interacting bosons. An added $\uparrow$ forms a spinon and much of its spectral 


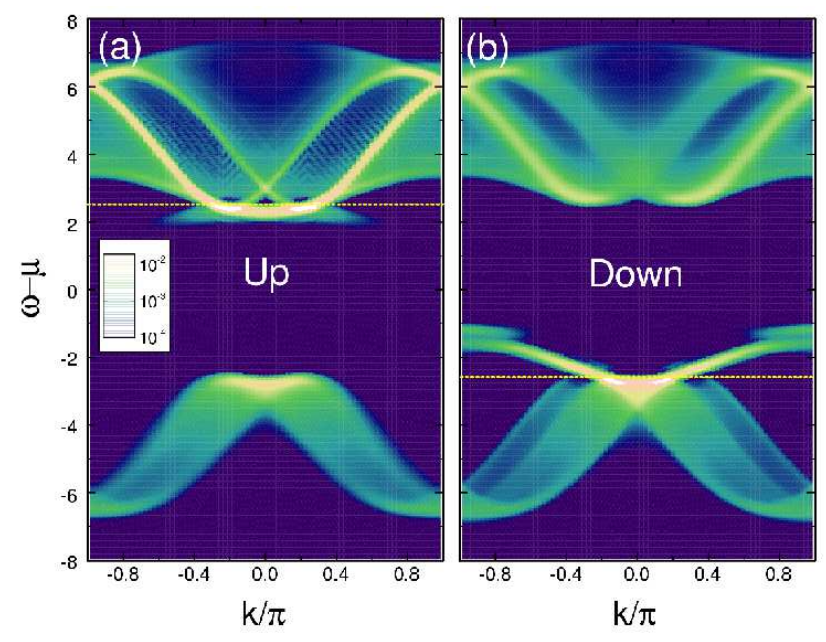

FIG. 2: (color online) "Photoemission" spectra for quarter-filled spin-polarized Hubbard chain, with $U=-8 t, N_{\uparrow}=24, N_{\downarrow}=16$. The energy scale has been chosen relative to the average chemical potential $\mu=\left(\mu_{\uparrow}+\mu_{\downarrow}\right) / 2$. The Fermi levels for each spin species are indicated by the horizontal lines (see text).

weight thus follows a spinon dispersion with bandwidth $4 t$. Since the wavefunction changes sign when the $\uparrow$ exchanges position with a 2 , the lowest-energy spinon states are at the momenta $\pm \pi / 4$ set by the density of the 2 's. However, the added fermion may also excite holon modes, and a careful look at the upper part of Fig. 1(c) reveals a continuum, with a weaker feature at the lower edge of the continuum which has a holon-like dispersion. This continuum arises when part of the added momentum is used to excite holon modes of the quasi-condensate.

Removing a fermion requires breaking a pair (a "2"), and this process apparently couples more strongly to the holon degrees of freedom, as can be seen by the flatter dispersion of the strong part of the spectrum at low momentum in the bottom part of Fig. 1(c). However, at higher momentum, this PES spectrum, although much weaker, has a continuum with a mostly spinon-like dispersion. Here the process apparently removes a low-momentum pair from the quasi-condensate and makes a spinon, with the spinon taking most of the momentum. At half-filling, there is particle-hole symmetry and the PES and IPES spectra are thus equivalent, both containing strong spinon and holon signals. [29]

We now turn our attention to the single-fermion spectrum in the polarized case, shown in Fig. 2. where we took $N_{\uparrow}-N_{\downarrow}=$ 8. Since the system is no longer symmetric under timereversal, the spectral functions for the up and down fermions are different. Correspondingly, we can determine the chemical potential of each species: $\mu_{\sigma}=\left[E\left(N_{\sigma}+1\right)-E\left(N_{\sigma}-\right.\right.$ 1)]/2. The average chemical potential is $\mu=\left(\mu_{\uparrow}+\mu_{\downarrow}\right) / 2$, while the effective Zeeman field is $h=\left(\mu_{\uparrow}-\mu_{\downarrow}\right) / 2$. We find it instructive to plot the spectra with energies relative to $\mu$. Note that $\mu$ is still in the "pairing" gap, but now $\mu_{\uparrow}$ is in the band above the gap, while $\mu_{\downarrow}$ is in the band below the gap.
At this fairly large $|U|$, we can describe this system as a quasi-condensate of bosonic 2's with density $k_{F \downarrow} / \pi=1 / 5$ and momentum $\pm Q= \pm \pi / 10$, plus a density $Q / \pi=1 / 10$ of excess unpaired $\uparrow$ 's. The 2's are bound pairs and sit below the gap and just below $\mu_{\downarrow}$. In the PES spectrum one can remove a fermion of either spin from one of these singlet pairs; these are the strong low-momentum features near $\omega-\mu \cong-3$. The weaker bands dispersing strongly to lower energy from these features arise from removing one member of a pair and leaving the other member in a spinon-like state.

The excess unpaired $\uparrow$ 's lie at energy just below $\mu_{\uparrow}$ and can be seen there in the spin-up PES spectrum. The wavefunctions of the $\uparrow$ 's change sign on passing each 2; as a result the lowest energy states of the corresponding spinon-like modes are at $\pm k_{F \downarrow}$; it is near these momenta where the up PES intensity is largest. The strongest bands in the up spectrum cross $\mu_{\uparrow}$ at $\pm k_{F \uparrow}$, just as in the noninteracting system. But one can also see weaker bands crossing $\mu_{\uparrow}$ at $\pm\left(2 k_{F \downarrow}-k_{F \uparrow}\right)$, which correspond to three-particle excitations in the noninteracting system. At low momentum and energies below $\mu_{\uparrow}$ there is a fairly flat holon-like dispersion of the up spectral weight; presumably here the excitation also transfers some momentum to the (heavy) pairs.

In the spin-down IPES spectrum, there is a heavy holonlike band at energies just above $\mu_{\downarrow}$. This arises from adding a down fermion that pairs with one of the excess up fermions with momentum $|k| \leq k_{F \uparrow}$, resulting in a pair (a 2 ) which carries most of the added momentum. This feature in the IPES is strong only for $|k| \geq k_{F \downarrow}$, since the down-spin states at lower momentum than this are already occupied. This band continues to the PES spectrum below $\mu_{\downarrow}$, crossing the chemical potential at $k= \pm k_{F \downarrow}$, as in the noninteracting system. At the zone boundary this holon-like band splits in to two faint features at $(\omega-\mu)$ near -1 and -1.5 , for reasons we do not yet understand. In the PES spectrum there are also weaker features approaching $\mu_{\downarrow}$ at momenta $\pm\left(2 k_{F \uparrow}-k_{F \downarrow}\right)$, which again correspond to three-particle excitations in the noninteracting system.

In the IPES spectrum above the gap, we can see that for both spins the added particle can excite a continuum of states with both spinon-like and holon-like dispersions. The sharpest feature is a spinon band, which is substantially sharper in the up IPES than in the down. The general appearance of this part of the IPES spectral weight is similar to that of the unpolarized case in Fig.1(c).

In order to examine the quasi-condensate and the pair-like excitations, we calculated the spectral weight of removing a pair $b_{i}=c_{i \uparrow} c_{i \downarrow}$, shown in Fig. 3(c) and (d). For the unpolarized case, we see a large concentration of spectral weight at zero energy and zero momentum in the PES, due to the zeromomentum quasi-condensate. In the polarized case, the zeroenergy spectral weight of this quasi-condensate splits into two features at $k= \pm Q= \pm\left(k_{F \uparrow}-k_{F \downarrow}\right)$, as expected for this FFLO-like state.

In Fig. 3 a) we show the dynamical structure factor for the density operator in the polarized case. One noteworthy differ- 


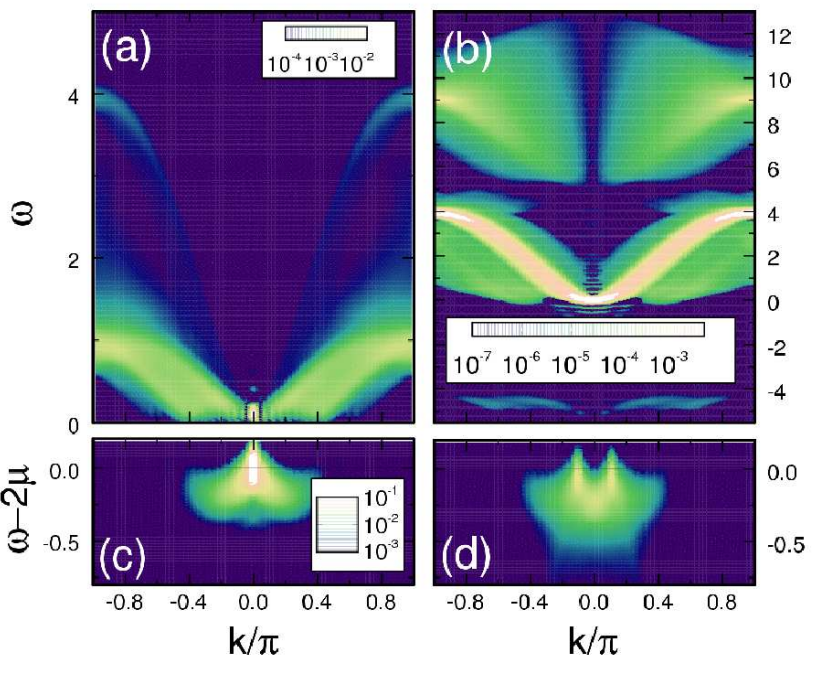

FIG. 3: (color online) Dynamical structure factors of the (a) "charge" density $n(k, \omega)$, and (b) spin lowering operator $S_{-}(k, \omega)$ for the polarized, quarter-filled Hubbard chain of Fig. 2. (c) Spectral weight for removing a pair from the unpolarized system, and (d) the polarized system. In (c) and (d) the energy scale is relative to the chemical potential of a pair $2 \mu$.

ence from the unpolarized case is the appearance of a weak spinon-like feature. This occurs because in this polarized system away from half-filling, the spinon-like mode is no longer purely spin so couples to the density.

Since time-reversal symmetry is broken by the spin polarization, the response functions for the spin operators $S_{z}, S_{+}$, and $S_{-}$are now all different. The structure factor for $S_{z}$ (not shown) exhibits, besides the excitations across the gap present in the unpolarized system (Fig. 1(b)), a gapless band that originates from spin excitations within the bands that cross the Fermi surfaces. The operator $S_{+}$flips spins up, breaking pairs, so its spectrum only shows excitations across the gap. The richest of these spin dynamical functions is $S_{-}(k, \omega)$, shown in Fig 3 (b). The action of the operator $S_{-}$on the ground state can cause three possible outcomes that each occupy a separate energy window: 1) it can break a pair; 2) it can flip an unpaired $\uparrow$ to an unpaired $\downarrow$, making a gapless spin fluctuation; or 3) the flipped spin can pair with another unpaired $\uparrow$ and be absorbed by the quasi-condensate. We find that the spectral weight for this last process is very weak, but detectable; it is visible just below energy -4 in Fig. 3b. We believe this very small weight is due to the product of two small factors: the low probability (due to fermionic antisymmetry) that two unpaired $\uparrow$ 's are on adjacent lattice sites before one of them is flipped down, and the low overlap between the resulting state after flipping, and the ground state with a bound pair, since the latter mostly consists of doubly-occupied sites.

To summarize, we have reported and discussed the rich features of the particle, pair, spin and density spectral weights for the quasi-FFLO superfluid ground state of a partially spinpolarized fermionic Hubbard chain with attractive interac- tions. We have found that a rigorous treatment, particularly to describe properties involving excitations, should still rely on the Luttinger liquid picture.

AEF is grateful to F. Heidrich-Meisner, M. Troyer, M.P.A. Fisher, and C. Nayak for useful and stimulating discussions. This work was partially supported (DAH) under ARO Award W911NF-07-1-0464 with funds from the DARPA OLE Program.

[1] F.D.M. Haldane, J. Phys. C: Solid State Phys. 14, 2585 (1981); Phys. Rev. Lett. 47, 1840 (1981).

[2] J. Voit, Rep. Prog. Phys. 58, 977 (1995).

[3] T. Giamarchi, "Quantum physics in one dimension", Oxford University Press (2004).

[4] T. Lorenz, et al., Nature 418, 614 (2002).

[5] P. Segovia, et al., Nature 402, 504 (1999); R. Losio, et al., Phys. Rev. Lett. 86, 4632 (2001).

[6] M. Bockrath, et al., Nature 397, 598 (1999).

[7] O.M. Auslaender, et al., Science 308, 88 (2005).

[8] A. Recati, et al., Phys. Rev. Lett. 90, 020401 (2003).

[9] L. Kecke, H. Grabert, and W. Häusler, Phys. Rev. Lett. 94, 176802 (2005).

[10] K. Penc and J. Sólyom, Phys. Rev. B 47, 6273 (1993).

[11] H. Frahm and T. Vekua, arXiv:0710.4498.

[12] C. Kollath and U. Schollwöck, New. J. of Phys., 8, 220 (2006).

[13] V.J. Emery, Phys. Rev. B 14, 2989 (1976).

[14] K. Yang, Phys. Rev. B 63, 140511(R) (2001).

[15] P. Fulde and A. Ferrell, Phys. Rev. 135, A550 (1964).

[16] A. Larkin and Y.N. Ovchinnikov, Zh. Eksp. Teor. Fiz. 47, 1136 (1964) [Sov. Phys. JETP 20, 762 (1965)].

[17] A. E. Feiguin and F. Heidrich-Meisner, Phys. Rev. B 76, 220508R (2007).

[18] M. Tezuka and M. Ueda, Phys. Rev. Lett. 100, 110403 (2008).

[19] G.G.Batrouni et al., Phys. Rev. Lett. 100, 116405 (2008).

[20] C. Kollath, W. Zwerger, and U. Schollwöck, Phys. Rev. Lett. 95, 176401 (2005).

[21] C. Kollath, J. Phys. B: At. Mol. Opt. Phys, 39, S65 (2006).

[22] E.H. Lieb and F.Y. Wu, Phys. Rev. Lett. 20,1445 (1968).

[23] F. Woynarovich, Phys. Rev. Lett. 43, 11448(1991).

[24] F. Woynarovich and K. Penc, Z. Phys. B 85,269 (1991).

[25] H. J. Schulz, cond-mat/9302006

[26] F. Essler, et al., "The One-Dimensional Hubbard Model”, Cambridge University Press (2005).

[27] N. Andrei, cond-mat/9408101

[28] K. Penc, et al., Phys. Rev. Lett. 77, 1390 (1996).

[29] H. Benthien, F. Gebhard, and E. Jeckelmann, Phys. Rev. Lett. 92, 256401 (2004).

[30] J. Favand, et al., Phys. Rev. B 55, R4859 (1997).

[31] S. R. White and A. E. Feiguin, Phys. Rev. Lett. 93, 076401 (2004).

[32] A. J. Daley, et al., J. Stat. Mech. : Theor. Exp., P04005 (2004).

[33] We typically used 400 DMRG states, keeping the truncation error $<10^{-7}$, and a 3rd-order Suzuki-Trotter expansion of the evolution operator with time-step $\tau=0.02$ or less (in units where the hopping is $t=1$ ). We calculated the time evolution up to time 30, and Fourier transformed using a cosine window.

[34] S. R. White and I. Affleck, arXiv:0712.3785

[35] M. Ogata, T. Sugiyama, and H. Shiba, Phys. Rev. B 43, 8401 (1991). 